\title{
Publisher Correction: Global plant-symbiont organization and emergence of biogeochemical cycles resolved by evolution-based trait modelling
}

Mingzhen Lu (D) and Lars O. Hedin

Correction to: Nature Ecology \& Evolution https://doi.org/10.1038/s41559-018-0759-0, published online 21 January 2019.

In the original PDF version of this Article, in Box 1 there were superfluous closing and opening parentheses in equation (1b), before and after the $F_{\mathrm{N}}$ term; the equation should have been as shown below.

$$
g_{i}=\omega_{i}(1-\alpha)\left(\frac{S_{i} A_{i}}{A_{i}+c_{i}^{S}}+\frac{M_{i} O_{i}}{O_{i}+c_{i}^{M}}+F_{\mathrm{N}}+\frac{v_{i}(X) A_{i}}{A_{i}+c_{i}^{v}}\right)
$$

This has now been corrected; the HTML version was unaffected.

Published online: 26 May 2020

https://doi.org/10.1038/s41559-020-1228-0

() The Author(s), under exclusive licence to Springer Nature Limited 2020 